\title{
Effect of Dominant Shade Trees on Coffee Production in Manasibu District, West Oromia, Ethiopia
}

\author{
Likassa Ebisa \\ Department of Natural Resources, College of Agriculture and Natural Resource, Wollega University, \\ Shambu Campus, Post Box No: 38 Shambu, Ethiopia
}

\begin{tabular}{|c|c|}
\hline \multirow{15}{*}{$\begin{array}{l}\text { Effect of shade trees species on coffee production in Manasibu district, West Ethiopia was } \\
\text { assessed by the current study. It was aimed to (i) identify the most suitable dominant shade } \\
\text { tree species for coffee production; (ii) assess the status of coffee production under different } \\
\text { dominant coffee shade trees and unshaded areas and (iii) evaluate the relationship of top soil } \\
\text { properties with coffee production in coffee based Agroforestry system. In this study, } \\
\text { randomized complete block design with three replications was used for data collection. } 48 \\
\text { circular samples were taken from areas around four dominant coffee shade tree species at } 3 \text {, } \\
5 \text { and } 7 \mathrm{~m} \text { from tree trunk and unshaded zone. There was no observed significant difference } \\
\text { in coffee production between shaded and unshaded zone of coffee farms. However, among } \\
\text { the species, coffee bean yield ( } 3.1 \mathrm{Mg} \text { ha }^{-1} \text { ) was observed significantly higher under Croton } \\
\text { macrostachyus than the others. There were significant positive correlations between coffee } \\
\text { weight and soil acidity ( } \mathrm{r}=0.44135 \text { ), between coffee weight and electric conductivity } \\
\text { (r=0.34302), between coffee weight and soil moisture content ( } \mathrm{r}=0.44738 \text { ). The influence of } \\
\text { top soil plant nutrients on coffee production is not linear. Available Potassium was not } \\
\text { supposed to influence coffee yield since highest reading was observed with lowest yield. It } \\
\text { can be possible to conclude that coffee based Agroforestry system is the better production } \\
\text { system when there is no intensive agronomic managements. Soil acidity status and moisture } \\
\text { content have significant effect on coffee production than top soil macro nutrients. If choosing } \\
\text { is needed, it appears to be a better option selecting C. macrostachyus than another dominant } \\
\text { shade tree species for higher coffee production in shade coffee production system due to its } \\
\text { coffee favorable environmental conditions (having normal soil pH and good soil moisture } \\
\text { content). }\end{array}$} & \\
\hline & Received : 03-06-2014 \\
\hline & Revised \\
\hline & ed : 26 \\
\hline & \\
\hline & \\
\hline & yus \\
\hline & erty \\
\hline & \\
\hline & \\
\hline & \\
\hline & \\
\hline & \\
\hline & \\
\hline & \\
\hline
\end{tabular}

\section{INTRODUCTION}

Coffee is a perennial woody shrub native to the understory of Ethiopian Afromontane rain forests between 1,000 and 2,000 m.a.s.I (Muleta et al., 2011; Reichhuber and Requate, 2012). Coffee production has attracted considerable attention globally, due to its economic, social, and ecological importance (Ferrell and Cockerill, 2012). The ecological services that provided by shade coffee production has generated much attention from scientific as well as other social interests due that only focusing on economic growth in the earlier resulted to the biodiversity loss (Reichhuber and Requate, 2012). Coffee cultivation systems fall along a continuum, ranging from traditionally grown under the canopy of shade trees to the industrial plantations with little or no shade. Accordingly, in Ethiopia, coffee has been cultivating mainly in traditional method (Bote and Struik, 2011) in coffee based Agroforestry system.

Coffee ranks among the five most valuable agricultural exports from developing nations (Ricketts et al., 2004) and the major source of foreign currency earnings as well as a significant proportion of tax income and gross domestic product for most of coffee producing countries. Among the 25 coffee producers on the African continent, Ethiopia is the first largest producer and the fifth of the world after Brazil, Vetinam, Indonesia and Colombia (AfDB, 2010). Although there is a growing worldwide movement to support and enhance organic coffee production systems under shade trees, study on the effects of shade on growth, production and quality of coffee is very scarce (Bote and Struik, 2011). Thus, studies to determine the relationship between shade trees and coffee yields on farm have not been conducted well.

Among tree species found in different coffee farms of Ethiopia, only few shade tree species are abundantly preferred both in the smallholder coffee farms and in large scale plantations. The retaining or planting of trees in the coffee farms is based on the understanding that it provides better condition for coffee plants in reducing excessive light, mulch the soil with litter (Beer et al., 1998), reducing periodic over-bearing and subsequent die-back of coffee branches (Bote and Struik, 2011), fodder, timber and other products. But there is no enough 


\section{Likassa Ebisa}

study conducted in Ethiopia which specifically indicates the effect of shade trees on coffee shrub density as well as yield. Therefore, this study was intended with identifying the most important dominant shade tree species which may contribute more for maximum and healthy coffee production as recommended by the study of Ebisa (2012) after identifying the dominant coffee shade tree species from west Oromia. The study also investigated factors which primarily affect coffee production within shade coffee production system. Generally, the scope of the study is to improve the product and productivity of shade coffee farms. Therefore, the study was aimed to assess the effect of dominant shade trees on coffee production in coffee based Agroforestry system of Manasibu district.

\section{MATERIALS AND METHODS}

\section{Site Description}

The study was conducted in Manasibu districts, western Oromia, Ethiopia (Figure 1). Manasibu consists of mid altitude (68\%) and lowland (32\%) and located at $09^{\circ}$ $48^{\prime} \mathrm{N}$ and $35^{\circ} 06^{\prime} \mathrm{E}$. Accordingly, the altitudinal variation ranges from 1249 to 1933 m.a.s.I. Mean annual temperature varies from 19 to $22^{\circ} \mathrm{C}$. The mean annual rainfall is $950 \mathrm{~mm}$. Fluvisols, Regosols, and Vertisols are the three soil types of the study area. The area is characterized by crop-livestock mixed farming system. Coffee plantation is the major one in sustaining the life of most residents. Guyo Sachi Lafto Salga (G/S/L/Salga) was the village selected for conducting the study. $\mathrm{G} / \mathrm{S} / \mathrm{L} / \mathrm{Salga}$ has relatively good access to asphalt road and found at about $17 \mathrm{~km}$ from Mendi town. Household $(\mathrm{HH})$ dependency on coffee production is high in this village.

\section{Sample Design and Data Collection}

Single village was selected purposefully among the others because of its' first level in the coffee producing villages of the district and its' affinity to asphalt road to town market. Coffee farms were selected during a preliminary survey within the selected village. Randomized complete block design with three replications was used for data collection from coffee farms having
Sci. Technol. Arts Res. J., July-Sep 2014, 3(3): 18-22

similar management practices, elevation and slope. Targeted trees (Cordia africana; Croton microstachyus; Albizea gummifera; Acacia abyssinica having dbh $\geq 10 \mathrm{~cm}$ and height of $\geq 10 \mathrm{~m}$ ) were selected randomly. Three circular plots were employed around the tree at $3 \mathrm{~m}(28.26$ $\left.\mathrm{m}^{2}\right), 5 \mathrm{~m}\left(78.5 \mathrm{~m}^{2}\right)$, and $7 \mathrm{~m}\left(153.86 \mathrm{~m}^{2}\right)$ distance from tree trunk following Bargali et al., (2009). Similarly, twelve sample $\left(28.26 \mathrm{~m}^{2}\right.$ ) plots (one each adjacent to shade trees) were sampled from an open area (unshaded) for comparison.

Data was collected from all forty eight (48) sample plots in which 36 and 12 were sampled from shaded zone and unshaded zones, respectively. Coffee shrubs abundance was quantified and changed to hectare basis. Four coffee plants (one from each perpendicular rays) were randomly selected to collect coffee berries on top, middle, and bottom branches following Soto-Pinto et al. (2000); Bote and Struik (2011) at the above defined space from tree trunk. Coffee determined by the bright red color (fully ripened) harvested and dried until a constant weight was reached (moisture content 12\%). Dried coffee bean without exocarb was measured using digital measuring balance. Extracted and dried coffee was measured on 1000 seed weight base ( $\mathrm{g}$ per 1000 beans) for coffee weight under each treatment. Similarly, Coffee bean yield ( $\mathrm{Mg} \mathrm{ha}^{-1}$ ) was calculated following Siles et al. (2010) and Bote and Struik (2011). Yield data was extrapolated to yield per hectare.

Coffee yield= Number of fruits per tree * Weight of the fruit

Where, Number of fruits per tree $=$ Fruiting nodes * Number of fruits per node

Four top soil (15 cm depth) samples were taken from all canopy rays and mixed to give one representative sample. The mixed soil was air dried, sieved through a 2 $\mathrm{mm}$ sieve and analyzed in Nekemte soil laboratory (Oromia Agricultural Research Institute) to identify soil $\mathrm{pH}$, Electric conductivity (EC), Soil organic matter (SOM), Phosphorus $(P)$, Potassium $(K)$, Nitrogen $(N)$, and Soil moisture content (SMC).

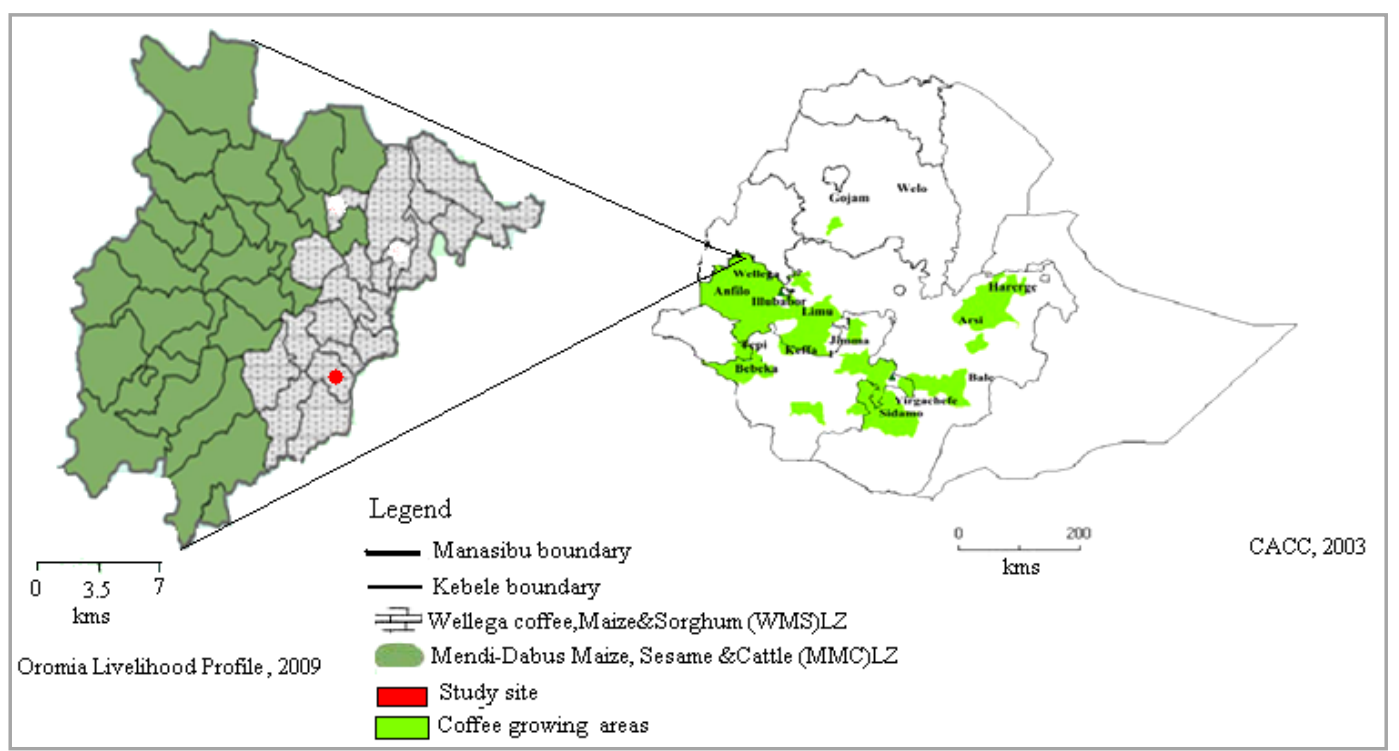

Figure 1: Location of the study area (Manasibu district) from administrative map of Ethiopia showing the main coffee growing areas 


\section{Likassa Ebisa}

\section{Data Analysis}

Coffee shrub density, coffee bean yield, coffee weight, and soil property parameters were compared between shaded and open areas as well as among the shade tree species. Effect of some soil physical and chemical properties on coffee shrub density, weight and yield was computed. Analysis of variance has been used to test the differences. Means were separated using the Tukey's (HSD) comparison test at $(P<0.05)$. Correlation analyses were also used to detect relationships among coffee shrub density, coffee weight, and soil parameters. All the analyses were carried out using a statistical analysis system (SAS Institute Inc., 2002).

\section{RESULTS AND DISCUSSION \\ Coffee Weight}

There was observed relative higher coffee weight (214.64 $\mathrm{g} / 1000$ bean) and coffee bean yield $\left(2.59 \mathrm{Mg} \mathrm{ha}^{-1}\right)$
Sci. Technol. Arts Res. J., July-Sep 2014, 3(3): 18-22

in shaded zone than unshaded zone of coffee farms although the difference was not statistically significant (Table 1). There was similar report from Jima zone of Ethiopia (Bote and Struik, 2011). Finding of Geromel et al. (2008) indicated that coffee weight was significantly higher in shade zone. Earlier study of Muleta et al. (2011) from southwest Ethiopia also confirm higher coffee yield from shade grown. Contrarily, coffee bean yield was reported to be relatively higher in unshaded coffee zone (Bote and Struik, 2011). Coffee based Agroforestry system would moderate extremes of high temperature, thereby creating a more adequate microclimate for coffee production than full sun coffee (Souza et al., 2012; Bote and Struik, 2011and Siebert, 2002). According to Bote and Struik (2011), shade resulted in heavier and larger coffee beans mainly due to its effect on temperature and the duration of the ripening period.

Table 1: Coffee yield variables, coffee density and soil (at 0-15 cm depth) under shaded and unshaded coffee zones of Manasibu district, West Ethiopia

\begin{tabular}{|c|c|c|c|}
\hline \multirow{2}{*}{ Variables } & \multicolumn{2}{|c|}{ Treatment } & \multirow{2}{*}{$P$-value } \\
\hline & Shaded zone & Unshaded zone & \\
\hline Coffee weight ( $\mathrm{g} / 1000$ beans) & $214.6( \pm 37.5)^{\mathrm{a}}$ & $171.00( \pm 6.5)^{\mathrm{a}}$ & 0.053 \\
\hline Coffee bean yield (Mg/ha) & $2.6( \pm 0.96)^{a}$ & $2.3( \pm 0.04)^{a}$ & 0.631 \\
\hline Coffee shrub density (plant ha ${ }^{-1}$ ) & $8649.8( \pm 9413.5)^{a}$ & $4566.5( \pm 397.5)^{\mathrm{a}}$ & 0.150 \\
\hline$\% \mathrm{TN}$ & $0.44( \pm 0.12)^{a}$ & $0.41( \pm 0.07)^{a}$ & 0.652 \\
\hline$\% \mathrm{OM}$ & $8.86( \pm 2.44)^{a}$ & $8.17( \pm 1.31)^{a}$ & 0.635 \\
\hline $\mathrm{P}(\mathrm{ppm})$ & $5.44( \pm 9.53)^{a}$ & $0.00( \pm 0.00)^{\mathrm{a}}$ & 0.335 \\
\hline $\mathrm{K}(\mathrm{mg} / \mathrm{Kg})$ & $3.15( \pm 1.85)^{a}$ & $3.08( \pm 2.65)^{a}$ & 0.954 \\
\hline $\mathrm{pH} 1: 2.5(\mathrm{~W} / \mathrm{V})$ in water & $5.95( \pm 0.37)^{a}$ & $5.64( \pm 0.25)^{a}$ & 0.171 \\
\hline $\mathrm{EC}(\mathrm{mS} / \mathrm{cm})$ & $0.09( \pm 0.14)^{a}$ & $0.05(0.02)^{a}$ & 0.623 \\
\hline SMC & $2.40( \pm 1.09)^{\mathrm{a}}$ & $2.11( \pm 0.12)^{a}$ & 0.654 \\
\hline
\end{tabular}

Means $( \pm S D)$ followed by the same letter horizontally in the row are not significant different by Tukey's HSD $(P=0.05)$

There is no significant difference in coffee weight and bean yield among the dominant shade trees. However, higher coffee weight ( $237.99 \mathrm{~g} / 1000$ bean) and bean yield (3.09 $\mathrm{Mg} \mathrm{ha}^{-1}$ ) were observed under C. macrostachyus (Table 2). Similarly, earlier studies also confirm the relative higher yield under Millettia ferruginea (Aerts et al., 2011) and significantly highest under A. abyssinica (Kufa et al., 2007). Such variation may caused from soil organic matter, total nitrogen and organic carbon provided by the plants (Kufa et al., 2007; Aerts et al., 2011). C. macrostachyus leads coffee production to be better due to having good litter. Similarly, Albizia schimperiana, $M$. ferruginea, A. abyssinica, E. abyssinica, Calpurinea subdecondra and C. africana (Kufa et al., 2007) and Inga densiflora (Siles et al., 2010) were preferred for coffee production due to their contributions in modification of the microclimate.

Table 2: Soil (at 0-15 cm depth), coffee yield variables and coffee density among shade tree species under shaded zones in Manasibu district, West Ethiopia

\begin{tabular}{|c|c|c|c|c|c|}
\hline \multirow[b]{2}{*}{ Variables } & \multicolumn{4}{|c|}{$\begin{array}{c}\text { Treatment } \\
\end{array}$} & \multirow[b]{2}{*}{$P$-value } \\
\hline & Cordia africana & $\begin{array}{c}\text { Croton } \\
\text { macrostachyus }\end{array}$ & $\begin{array}{c}\text { Albizea } \\
\text { gummifera }\end{array}$ & $\begin{array}{c}\text { Acacia } \\
\text { abyssinica }\end{array}$ & \\
\hline Coffee weight (g/1000 beans) & $198.5( \pm 39.0)^{\mathrm{a}}$ & $237.9( \pm 28.6)^{a}$ & $203.3( \pm 39.5)^{\mathrm{a}}$ & $218.7( \pm 34.2)^{\mathrm{a}}$ & 0.101 \\
\hline Coffee bean yield $(\mathrm{Mg} / \mathrm{ha})$ & $2.2( \pm 0.6)^{\mathrm{b}}$ & $3.1( \pm 1.2)^{a}$ & $2.0( \pm 0.6)^{\mathrm{b}}$ & $3.0( \pm 0.8)^{a}$ & 0.019 \\
\hline Coffee shrub density $\left(\mathrm{ha}^{-1}\right)$ & $5583.2( \pm 1629.3)^{b}$ & $9439.9( \pm 9295.8)^{a}$ & $6057.5( \pm 4413.2)^{\mathrm{b}}$ & $9518.5( \pm 11080.3)^{a}$ & 0.008 \\
\hline$\% \mathrm{TN}$ & $0.38( \pm 0.103)^{\mathrm{a}}$ & $0.48( \pm 0.13)^{\mathrm{a}}$ & $0.44( \pm 0.12)^{\mathrm{a}}$ & $0.46( \pm 0.13)^{\mathrm{a}}$ & 0.361 \\
\hline$\% \mathrm{OM}$ & $7.71( \pm 2.05)^{\mathrm{a}}$ & $9.70( \pm 2.59)^{\mathrm{a}}$ & $8.79( \pm 2.38)^{a}$ & $9.22( \pm 2.66)^{a}$ & 0.365 \\
\hline $\mathrm{P}(\mathrm{ppm})$ & $10.20( \pm 13.34)^{\mathrm{a}}$ & $10.20(10.55)^{a}$ & $1.36( \pm 2.28)^{b}$ & $0.00( \pm 0.00)^{b}$ & 0.021 \\
\hline $\mathrm{K}(\mathrm{mg} / \mathrm{Kg})$ & $4.98( \pm 2.07)^{\mathrm{a}}$ & $3.20( \pm 1.41)^{b}$ & $1.59( \pm 0.92)^{b c}$ & $2.81( \pm 1.11)^{\mathrm{c}}$ & 0.000 \\
\hline $\mathrm{pH} 1: 2.5(\mathrm{~W} / \mathrm{V})$ in water & $6.24( \pm 0.28)^{\mathrm{a}}$ & $6.25( \pm 0.22)^{a}$ & $5.54( \pm 0.21)^{c}$ & $5.77( \pm 0.14)^{b}$ & $<0.000$ \\
\hline $\mathrm{EC}(\mathrm{mS} / \mathrm{cm})$ & $0.093( \pm 0.02)^{a}$ & $0.160( \pm 0.27)^{\mathrm{a}}$ & $0.066( \pm 0.00)^{\mathrm{a}}$ & $0.049( \pm 0.01)^{\mathrm{a}}$ & 0.341 \\
\hline SMC & $2.041( \pm 0.00)^{\mathrm{a}}$ & $3.242( \pm 1.91)^{\mathrm{a}}$ & $2.041( \pm 0.00)^{\mathrm{a}}$ & $2.277( \pm 0.71)^{\mathrm{a}}$ & 0.053 \\
\hline
\end{tabular}

Across the distance from tree trunk, there was observed significant difference in coffee weight under $C$. macrostachyus and $A$. gummifera. On the other hand, there was no linear change in under $C$. africana and $A$. abyssinica (Table 3 ). The increase of coffee weight closing to tree base indicates the enhancing property of the tree for production than those makes either decreasing or inconsistent. Contrarily, grain yield increase as distance increases from tree base and even maximum in the unshaded area of Acacia nilotica-based traditional Agroforestry system (Bargali et al., 2009). 
Table 3: Coffee yield variables, soil (at $0-15 \mathrm{~cm}$ depth) and coffee density across the distance from tree trunk under shade tree species in Manasibu district, West Ethiopia

\begin{tabular}{|c|c|c|c|c|c|c|c|c|c|c|c|}
\hline \multirow[b]{2}{*}{ Treatment } & \multirow[b]{2}{*}{ 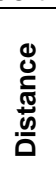 } & \multicolumn{10}{|c|}{ Variables } \\
\hline & & 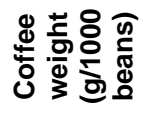 & 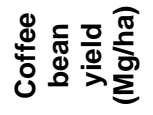 & 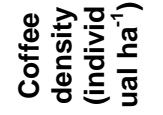 & $\% \mathrm{TN}$ & $\%$ OM & $P(p p m)$ & $\begin{array}{l}\mathrm{K}(\mathrm{mg} / \\
\mathrm{Kg})\end{array}$ & $\begin{array}{c}\mathrm{pH} 1: 2.5 \\
(\mathrm{~W} / \mathrm{V}) \text { in } \\
\text { water }\end{array}$ & $\begin{array}{c}\mathrm{EC} \\
(\mathrm{mS} / \mathrm{cm})\end{array}$ & SMC \\
\hline \multirow{3}{*}{$\begin{array}{c}\text { Cordia } \\
\text { africana }\end{array}$} & 1 & $\begin{array}{c}203.51 \\
( \pm 25.41)^{\mathrm{ab}}\end{array}$ & $\begin{array}{c}2.56 \\
( \pm 0.91)^{a}\end{array}$ & $\begin{array}{c}4666.90 \\
( \pm 204.30)^{c d}\end{array}$ & $\begin{array}{c}0.31 \\
( \pm 0.08)^{a}\end{array}$ & $\begin{array}{c}6.29 \\
( \pm 1.69)^{a}\end{array}$ & $\begin{array}{c}8.84 \\
( \pm 15.34)^{a}\end{array}$ & $\begin{array}{c}4.25 \\
( \pm 1.33)^{a}\end{array}$ & $\begin{array}{c}5.99 \\
( \pm 0.28)^{\text {bca }}\end{array}$ & $\begin{array}{c}0.08 \\
( \pm 0.02)^{a}\end{array}$ & $\begin{array}{c}2.04 \\
( \pm 1.28)^{b}\end{array}$ \\
\hline & 2 & $\begin{array}{c}184.06 \\
( \pm 39.32)^{b}\end{array}$ & $\begin{array}{c}2.32 \\
( \pm 0.29)^{a}\end{array}$ & $\begin{array}{c}3053.10 \\
( \pm 73.55)^{d}\end{array}$ & $\begin{array}{c}0.39 \\
( \pm 0.10)^{\mathrm{a}}\end{array}$ & $\begin{array}{c}7.75 \\
( \pm 2.04)^{a}\end{array}$ & $\begin{array}{c}19.05 \\
( \pm 16.36)^{a}\end{array}$ & $\begin{array}{c}6.85 \\
( \pm 2.50)^{\mathrm{ab}}\end{array}$ & $\begin{array}{c}6.43 \\
( \pm 0.25)^{a}\end{array}$ & $\begin{array}{c}0.11 \\
( \pm 0.02)^{a}\end{array}$ & $\begin{array}{c}2.04 \\
( \pm 1.28)^{b}\end{array}$ \\
\hline & 3 & $\begin{array}{c}208.04 \\
( \pm 58.47)^{\mathrm{ab}}\end{array}$ & $\begin{array}{c}1.69 \\
( \pm 0.36)^{a}\end{array}$ & $\begin{array}{c}3029.64 \\
( \pm 129.99)^{d}\end{array}$ & $\begin{array}{c}0.46 \\
( \pm 0.10)^{a}\end{array}$ & $\begin{array}{c}9.10 \\
( \pm 1.95)^{a}\end{array}$ & $\begin{array}{c}2.72 \\
( \pm 2.35)^{a}\end{array}$ & $\begin{array}{c}3.84 \\
( \pm 1.03)^{\mathrm{ab}}\end{array}$ & $\begin{array}{c}6.30 \\
( \pm 0.10)^{\mathrm{ab}}\end{array}$ & $\begin{array}{c}0.09 \\
( \pm 0.01)^{a}\end{array}$ & $\begin{array}{c}2.04 \\
( \pm 1.28)^{b}\end{array}$ \\
\hline \multirow{3}{*}{$\begin{array}{c}\text { Croton } \\
\text { macrostachyus }\end{array}$} & 1 & $\begin{array}{l}272.83 \\
( \pm 9.67)^{a}\end{array}$ & $\begin{array}{c}2.23 \\
( \pm 0.72)^{a}\end{array}$ & $\begin{array}{c}4492.57 \\
( \pm 707.71)^{\mathrm{cd}}\end{array}$ & $\begin{array}{c}0.55 \\
( \pm 0.16)^{a}\end{array}$ & $\begin{array}{c}10.78 \\
( \pm 0.21)^{a}\end{array}$ & $\begin{array}{c}10.88 \\
( \pm 13.58)^{a}\end{array}$ & $\begin{array}{c}3.91 \\
( \pm 2.02)^{a b}\end{array}$ & $\begin{array}{c}6.44 \\
( \pm 0.04)^{a}\end{array}$ & $\begin{array}{c}0.35 \\
( \pm 0.46)^{a}\end{array}$ & $\begin{array}{c}5.64 \\
( \pm 1.28)^{a}\end{array}$ \\
\hline & 2 & $\begin{array}{c}232.48 \\
( \pm 0.47)^{a b}\end{array}$ & $\begin{array}{c}3.74 \\
( \pm 1.28)^{a}\end{array}$ & $\begin{array}{c}6338.99 \\
( \pm 152.43)^{b}\end{array}$ & $\begin{array}{c}0.54 \\
( \pm 0.10)^{a}\end{array}$ & $\begin{array}{c}11.06 \\
( \pm 3.30)^{\mathrm{a}}\end{array}$ & $\begin{array}{c}10.02 \\
( \pm 12.41)^{a}\end{array}$ & $\begin{array}{c}2.94 \\
( \pm 1.18)^{\mathrm{ab}}\end{array}$ & $\begin{array}{c}6.29 \\
( \pm 0.11)^{\mathrm{ab}}\end{array}$ & $\begin{array}{c}0.45 \\
( \pm 0.01)^{a}\end{array}$ & $\begin{array}{c}2.04 \\
( \pm 0.00)^{b}\end{array}$ \\
\hline & 3 & $\begin{array}{l}208.65 \\
( \pm 1.46)^{a b}\end{array}$ & $\begin{array}{c}3.29 \\
( \pm 1.36)^{a}\end{array}$ & $\begin{array}{c}8488.09 \\
( \pm 679.36)^{a}\end{array}$ & $\begin{array}{c}0.36 \\
( \pm 0.08)^{\mathrm{a}}\end{array}$ & $\begin{array}{c}7.25 \\
( \pm 1.55)^{a}\end{array}$ & $\begin{array}{c}9.52 \\
( \pm 10.27)^{a}\end{array}$ & $\begin{array}{c}2.75 \\
( \pm 1.14)^{\mathrm{ab}}\end{array}$ & $\begin{array}{c}6.02 \\
( \pm 0.21)^{\mathrm{bca}}\end{array}$ & $\begin{array}{c}0.08 \\
( \pm 0.02)^{\mathrm{a}}\end{array}$ & $\begin{array}{c}2.04 \\
( \pm 0.00)^{b}\end{array}$ \\
\hline \multirow{3}{*}{$\begin{array}{c}\text { Albizea } \\
\text { gummifera }\end{array}$} & 1 & $\begin{array}{c}218.89 \\
( \pm 48.86)^{\mathrm{ab}}\end{array}$ & $\begin{array}{c}1.59 \\
( \pm 0.59)^{\mathrm{a}}\end{array}$ & $\begin{array}{c}4666.90 \\
( \pm 204.29)^{c d}\end{array}$ & $\begin{array}{c}0.37 \\
( \pm 0.07)^{\mathrm{a}}\end{array}$ & $\begin{array}{c}7.44 \\
( \pm 1.59)^{\mathrm{a}}\end{array}$ & $\begin{array}{c}1.36 \\
( \pm 2.36)^{a}\end{array}$ & $\begin{array}{c}1.48 \\
( \pm 1.25)^{b}\end{array}$ & $\begin{array}{c}5.60 \\
( \pm 0.28)^{c}\end{array}$ & $\begin{array}{c}0.06 \\
( \pm 0.01)^{a}\end{array}$ & $\begin{array}{c}2.04 \\
( \pm 1.28)^{\mathrm{b}}\end{array}$ \\
\hline & 2 & $\begin{array}{c}176.64 \\
( \pm 1.28)^{b}\end{array}$ & $\begin{array}{c}1.75 \\
( \pm 0.28)^{a}\end{array}$ & $\begin{array}{c}5172.68 \\
( \pm 602.57)^{\mathrm{cb}}\end{array}$ & $\begin{array}{c}0.55 \\
( \pm 0.14)^{a}\end{array}$ & $\begin{array}{c}10.99 \\
( \pm 2.85)^{\mathrm{a}}\end{array}$ & $\begin{array}{c}2.04 \\
( \pm 3.53)^{a}\end{array}$ & $\begin{array}{c}1.69 \\
( \pm 1.20)^{b}\end{array}$ & $\begin{array}{c}5.56 \\
( \pm 0.29)^{c}\end{array}$ & $\begin{array}{c}0.07 \\
( \pm 0.00)^{\mathrm{a}}\end{array}$ & $\begin{array}{c}2.04 \\
( \pm 1.28)^{b}\end{array}$ \\
\hline & 3 & $\begin{array}{c}214.27 \\
( \pm 47.19)^{\mathrm{ab}}\end{array}$ & $\begin{array}{c}2.71 \\
( \pm 0.33)^{a}\end{array}$ & $\begin{array}{c}4332.94 \\
( \pm 187.62)^{d}\end{array}$ & $\begin{array}{c}0.39 \\
( \pm 0.04)^{\mathrm{a}}\end{array}$ & $\begin{array}{c}7.94 \\
( \pm 0.93)^{a}\end{array}$ & $\begin{array}{c}0.68 \\
( \pm 1.18)^{\mathrm{a}}\end{array}$ & $\begin{array}{c}1.60 \\
( \pm 0.60)^{b}\end{array}$ & $\begin{array}{c}5.47 \\
( \pm 0.04)^{c}\end{array}$ & $\begin{array}{c}0.06 \\
( \pm 0.01)^{a}\end{array}$ & $\begin{array}{c}2.04 \\
( \pm 1.28)^{b}\end{array}$ \\
\hline \multirow{3}{*}{$\begin{array}{c}\text { Acacia } \\
\text { abyssinica }\end{array}$} & 1 & $\begin{array}{c}191.44 \\
( \pm 0.09)^{a b}\end{array}$ & $\begin{array}{c}3.09 \\
( \pm 1.25)^{a}\end{array}$ & $\begin{array}{c}4430.99 \\
( \pm 707.71)^{\mathrm{cd}}\end{array}$ & $\begin{array}{c}0.56 \\
( \pm 0.15)^{a}\end{array}$ & $\begin{array}{c}11.27 \\
( \pm 3.05)^{a}\end{array}$ & $\begin{array}{c}0.00 \\
( \pm 0.00)^{a}\end{array}$ & $\begin{array}{c}2.41 \\
( \pm 1.11)^{\mathrm{ab}}\end{array}$ & $\begin{array}{c}5.86 \\
( \pm 0.07)^{\text {bca }}\end{array}$ & $\begin{array}{c}0.05 \\
( \pm 0.01)^{a}\end{array}$ & $\begin{array}{c}2.75 \\
( \pm 1.23)^{\mathrm{b}}\end{array}$ \\
\hline & 2 & $\begin{array}{l}212.50 \\
( \pm 3.38)^{a b}\end{array}$ & $\begin{array}{c}3.21 \\
( \pm 1.06)^{\mathrm{a}}\end{array}$ & $\begin{array}{c}6687.66 \\
( \pm 621.49)^{\mathrm{b}}\end{array}$ & $\begin{array}{c}0.44 \\
( \pm 0.13)^{a}\end{array}$ & $\begin{array}{c}8.73 \\
( \pm 2.52)^{a}\end{array}$ & $\begin{array}{c}0.00 \\
( \pm 0.00)^{\mathrm{a}}\end{array}$ & $\begin{array}{c}2.72 \\
( \pm 1.42)^{\mathrm{ab}}\end{array}$ & $\begin{array}{c}5.76 \\
( \pm 0.17)^{\mathrm{bc}}\end{array}$ & $\begin{array}{c}0.05 \\
( \pm 0.01)^{a}\end{array}$ & $\begin{array}{c}2.04 \\
( \pm 0.00)^{b}\end{array}$ \\
\hline & 3 & $\begin{array}{c}252.22 \\
( \pm 30.23)^{\mathrm{ab}}\end{array}$ & $\begin{array}{c}2.84 \\
( \pm 0.32)^{\mathrm{a}}\end{array}$ & $\begin{array}{c}9436.89 \\
( \pm 595.62)^{a}\end{array}$ & $\begin{array}{c}0.38 \\
( \pm 0.08)^{a}\end{array}$ & $\begin{array}{c}7.67 \\
( \pm 1.54)^{\mathrm{a}}\end{array}$ & $\begin{array}{c}0.00 \\
( \pm 0.00)^{\mathrm{a}}\end{array}$ & $\begin{array}{c}3.30 \\
( \pm 1.05)^{\mathrm{ab}}\end{array}$ & $\begin{array}{c}5.69 \\
( \pm 0.15)^{c}\end{array}$ & $\begin{array}{c}0.05 \\
( \pm 0.01)^{a}\end{array}$ & $\begin{array}{c}2.04 \\
( \pm 0.00)^{b}\end{array}$ \\
\hline Control & & $\begin{array}{c}170.95 \\
( \pm 6.50)^{\mathrm{b}} \\
\end{array}$ & $\begin{array}{c}2.31 \\
( \pm 0.04)^{a} \\
\end{array}$ & $\begin{array}{c}3566.54 \\
( \pm 397.54)^{d}\end{array}$ & $\begin{array}{c}0.41 \\
( \pm 0.07)^{a}\end{array}$ & $\begin{array}{c}8.17 \\
( \pm 1.31)^{a} \\
\end{array}$ & $\begin{array}{c}0.00 \\
( \pm 0.00)^{a}\end{array}$ & $\begin{array}{c}3.08 \\
( \pm 2.65)^{\mathrm{ab}}\end{array}$ & $\begin{array}{c}5.64 \\
( \pm 0.25)^{c} \\
\end{array}$ & $\begin{array}{c}0.05 \\
( \pm 0.02)^{a} \\
\end{array}$ & $\begin{array}{c}2.11 \\
( \pm 0.12)^{b}\end{array}$ \\
\hline$P$-value & & 0.0176 & 0.0595 & $<.0001$ & 0.0664 & 0.0683 & 0.1977 & 0.0210 & $<.0001$ & 0.3138 & $<.0001$ \\
\hline
\end{tabular}

\section{Coffee Density}

Coffee shrub was observed to be denser ( 8649.8 plant $\mathrm{ha}^{-1}$ ) in shaded zone (Table 1). It has been found similarly denser in shaded coffee zones from south Colombia (Bosselmann et al., 2009), whereas in Costa Rica, it was slightly higher in open zone (Silas et al., 2010). Coffee shrub density was significantly higher under A. abyssinica (9519 plant ha $^{-1}$ ) (Table 2). Similarly there was significant different across the distance from the truck (Table 3). Although coffee shrubs plantation has been performed in similar design; the difference in density may be caused from the relative suitable soil plant relationship in the shaded system. Additionally, shade makes the soil physical property to be maintained and enhanced the coffee plant to be viable and saved. The relationship between coffee weight and coffee shrub density $(r=$ $0.23381 ; P=0.1520$ ) is not linear. Increase or decrease of coffee weight is not from coffee plant density. However, following raise of plants density; production may decrease.

\section{Soil Factors Affecting Coffee Production}

Soil organic matter, soil pH, Electric conductivity and total Nitrogen, Phosphorus, and Potassium were higher in shaded zone although not significant (Table 1). Shaded system has relatively better chemical properties in total Nitrogen, total Carbon, Potassium, and Cation exchange capacity (Siles et al., 2010), soil organic matter and soil $\mathrm{Ph}$ (Bosselmann et al., 2009) than sun coffee farms. Inversely available Phosphorous, Potassium, and Cation exchange capacity were analyzed to be higher in open coffee areas; whereas, total nitrogen, total organic carbon were higher in shaded areas top soil (Souza et al., 2012). Lower soil $\mathrm{pH}$ had reported contrarily from shaded areas of the farm (Siles et al., 2010; Souza et al., 2012). As some of the soil chemical properties increasing in shaded coffee farms, coffee yield also raise up. Therefore, existing shade trees are contributing positively through improving the top soil chemical and physical property thereby affecting the production.

There is no significant difference in soil moisture content between shade and unshaded area although it is higher in shade coffee areas as expected. In line of the current study, Siles et al. (2010) has also discussed that there is no significant variation. This may indicate the reduction in soil temperature, observed under shade, which was mainly caused by the ability of shaded soil to stabilize the local thermal balances (Kufa et al., 2007; Siles et al., 2010; Bote and Struik, 2012).

Among the shade tree species, there is significant difference in available Phosphorus, available Potassium, and Soil $\mathrm{pH}$. Whereas, there is no significant difference in per cent total Nitrogen, per cent Organic matter, Electric conductivity, and soil moisture content. Higher total nitrogen per cent (0.48), Organic matter per cent (9.70), Available Phosphorus (10.20), soil pH (6.25), Electric conductivity (0.16), soil moisture content (3.242) were analyzed under C. macrostachyus. Higher Available Potassium (4.98) was from C. africana (Table 2). Inline of the current study the earlier finding of Aerts et al. (2011) from south west Ethiopia shown higher $\mathrm{pH}$, per cent $\mathrm{N}$ and Carbon from M. ferruginea; higher Cation exchange capacity and potassium from C. macrostachyus; higher Phosphorus from and A. gummifera, respectively.

In Ethiopia $A$. schimperiana, $M$. ferruginea, $A$. abyssinica, E. abyssinica, Calpurinea subdecondra and $C$. africana were seemed suitable coffee shade trees (Kufa et al., 2011). Accordingly, C. macrostachyus was found 


\section{Likassa Ebisa}

the most contributing tree from the current study area. Whereas it was estimated to be higher under the shade of A. abyssinica (Kufa et al., 2007) and M. ferruginea (Aerts et al., 2011) from south west Ethiopia. Their importance for coffee production has discussed in association to modification of the microclimate, decomposition from the litter fall in building soil fertility, intercepting half of the incoming light intensity (Kufa et al., 2011). Except, per cent soil organic matter and electric conductivity, other chemical properties of the soil and soil moisture content were increasing as closing to $C$. macrostachyus tree trunk, whereas such pattern was not observed from another shade tree species (Table 3).

Relationship of Soil Properties and Coffee Production

Tree crop interaction in coffee farms affects coffee production from above or below ground resource sharing. There was identified significant positive correlation between coffee weight and soil acidity $(r=0.44135 ; P=$ $0.0049)$, electric conductivity $(r=0.34302 ; P=0.0325)$, soil moisture content $(\mathrm{r}=0.44738 ; P=0.0043)$. However, the correlation between coffee weight and per cent of soil total nitrogen ( $\mathrm{r}=0.09817 ; P=0.5521)$, soil available phosphorus $(\mathrm{r}=0.11661 ; \quad P=0.4796)$, soil available Potassium $(r=0.10610 ; \quad P=0.5203)$ was not significant. Their relationship can be discussed as coffee production is more influenced by soil environmental amelioration formed by shade trees than soil macronutrients (N, P and $\mathrm{K})$ added. Coffee production has linear relationship with soil $\mathrm{pH}$. As soil acidity increases, coffee production decreases. Earlier study confirms the direct relationship of coffee yield or weight with soil acidity (Aerts et al., 2011). Similarly, as top soil moisture content increases coffee production also increases. The influence of top soil plant nutrients on coffee production is not linear. However, there was observed higher coffee yield in areas having relatively higher per cent total Nitrogen, Organic matter, and available Phosphorus. Whereas, available Potassium was not influential since coffee yield was less where it examined highest as also reported in Aerts et al. (2011).

\section{CONCLUSIONS}

With the absence of fertilizer application, irrigation, and agronomic practices, shade coffee production is better than coffee monoculture. C. macrostachyus was found the most important shade tree followed by $A$. abysinica, $A$. gummifera, and $C$. africana, respectively. The dominance of the species in the farm is mainly from the economic value as like $C$. africana rather than ecological services. Therefore, farmers should better considering the compatibility of shade tree species with coffee shrubs to harvest more coffee yield. C. macrostachyus was found the most important shade tree coffee production due to having quality litter, acid free soil and good soil moisture content facilitating higher yield. Soil acidity status and moisture content have significantly influence on coffee production to be higher or lower than top soil macro nutrients. As soil acidity decreases and moisture content increases, coffee production increases. There is no linear and consistent effect of Potassium and Phosphorus on coffee production. As distance increase from $C$. macrostachyus and $A$. abyssinica tree trunk, coffee production decreases. The relationship is not linear and consistent under $A$. gummifera and $C$. africana.

\section{REFERENCE}

Aerts, R., Hundera, K., Berecha, G., Gijbels, P., Baeten, M., Mechelena, M., Hermy, M., Muys, B., Honnay, O. (2011). Semi-forest coffee cultivation and the conservation of
Sci. Technol. Arts Res. J., July-Sep 2014, 3(3): 18-22

Ethiopian Afromontane rainforest fragments. Agriculture, Ecosystems and Environment 261:1034-1041.

African Development Bank (AfDB) (2010). Coffee Production in Africa and the Global Market Situation. Community Market Brief 1(2): 1-9.

Bargali, S.S., Kiran, B., Lalji, S., Lekha, G., Lakhera, M.L., (2009). Acacia nilotica-based traditional Agroforestry system: effect on paddy crop and management. Current Science 96(4): 581-587.

Beer, J., Muschler, R., Kass, D., and Somarriba, E. (1998). Shade management in coffee and cacao plantations. Agroforestry Systems 38:139-194.

Bosselmann, S.A., Dons K., Oberthur, T., Olsen, S.C., Raebild, A., Usma, H. (2009). The influence of shade trees on coffee quality in small holder coffee agroforestry systems in Southern Colombia. Agriculture, Ecosystems and Environment 129:253-260.

Bote, A.D., and Struik, P.C., 2011. Effect of shade on growth, production and quality of coffee (Coffea arabica) in Ethiopia. Journal of Horticulture \& Forestry 3(11):336-341.

Ebisa L. (2012). Tree species diversity in smallholder coffee farms of Western Ethiopia. LAP LAMBERT Academic Publishing, German, pp 76.

Ferrell, J., and Cockerill, K. (2012). Closing coffee production loops with waste to ethanol in Matagalpa, Nicaragua. Energy for Sustainable Development 16(1): 44-50.

Geromel, C., Ferreira, P.L., Davrieux, F., Guyot, B., Ribeyre, F., Scholz, S.B.M., Pereira, P.F., Vaast, P., Pot, D., Leroy, T., Filho, A.A., Vieira, E.G., Mazzafera, P., and Marraccini. (2008). Effects of shade on the development and sugar metabolism of coffee (Coffea Arabica) fruits. Plant Physiology and Biochemistry 46: 569-579.

Kufa, T., Yilma, A., Shimber, T., Netsere, A., Taye, E. (2007). Yield performance of Coffea arabicacultivars under different shade trees at Jimma Research Center, Southwest Ethiopia. In: Proceedings of the Second Intrl. Symp on Multi-strata Agroforestry Systems with Perennial Crops, Sep. 17-21, CATIE, Turrialba, Costa Rica.

Muleta, D., Assefa, F., Nemomissa, S., and Granhall, U. (2011). Socioeconomic benefits of shade trees in coffee production systems in Bonga and Yayu hurumu districts, southwestern Ethiopia: Farmers' perceptions. Ethiopian Journal of Education and Sciences 1:39-56.

Oromia Livelihood Profile (OLP). (2009). Oromiya livelihood zone Reports: Manasibu woreda west Wellega zone. Livelihood profile Oromia region, Ethiopia.

Reichhuber, A. and Requate, T. (2012). Alternative use systems for the remaining Ethiopian cloud forest and the role of Arabica coffee-A cost-benefit analysis. Ecological Economics 75:102-113.

Ricketts, T.H., Daily, G.C., Ehrlich, P. R., and Michener, C.D. (2004). Economic value of tropical forest to coffee production. PNAS 101:12579-12582.

Siles, P., Harmand J M., and Vaast, P. (2010). Effects of Inga densiflora on the microclimate of coffee (Coffea arabica L.) and overall biomass under optimal growing conditions in Costa Rica. Agroforestry Systems 78:269-286.

Soto-Pinto, L., Perfecto, I., Castillo-Hernandez, J., CaballeroNieto, J. (2000). Shade effect on coffee production at the northern Tzeltal zone of the state of Chiapas, Mexico. Agriculture, Ecosystems and Environment 80: 61-69.

Souza, N.H., Goedea, G.M, Brussaard, L., Cardoso, M.I., Duarteb, M., Gomes, C., Pulleman, M. (2012). Protective shade, tree diversity and soil properties in coffee agroforestry systems in the Atlantic Rainforest biome. Agriculture, Ecosystems and Environment 146:179-196. 